\title{
Histopathological and Molecular Features of a Conjunctival Caruncular Deep Penetrating Nevus
}

\author{
Jolique A. van Ipenburg a Jeffrey Damman ${ }^{a}$ Dion Paridaens ${ }^{b, c}$ \\ Robert M. Verdijk ${ }^{a}, b, d$
}

${ }^{a}$ Section of Ophthalmic Pathology, Department of Pathology, Erasmus MC - University Medical Center, Rotterdam, The Netherlands; ${ }^{\mathrm{b}}$ The Rotterdam Eye Hospital, Rotterdam, The Netherlands; ${ }^{\mathrm{C}}$ Department of Ophthalmology,

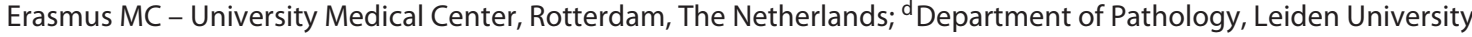
Medical Center, Leiden, The Netherlands

\section{Established Facts}

- Already known fact 1: Deep penetrating nevus (DPN) is a well-established histopathological and molecular distinct nevus subtype of the skin.

- Already known fact 2: Overlapping clinical and histopathological features might complicate distinguishing benign from malignant melanocytic lesions.

\section{Novel Insights}

- New information 1: Molecular analysis might be necessary in rendering a correct diagnosis; in this case, a DPN was confirmed by the presence of a $\beta$-catenin mutation with exclusion of molecular abnormalities as found in melanoma.

- New information 2: The presentation of a DPN of the lacrimal caruncle emphasizes the similarities of the caruncle with the skin.

\section{Keywords \\ Conjunctiva - Caruncle - Deep penetrating nevus - BRAF . $\beta$-Catenin}

\begin{abstract}
We describe the first presentation of a deep penetrating nevus (DPN) on the lacrimal caruncle. This lesion was seen in an 18-year-old woman presenting with hemorrhage of a longstanding pigmented mass on the caruncle. Histology showed a combined melanocytic neoplasm that consisted of two dif-
\end{abstract}

ferent melanocytic components. The differential diagnosis, based on histological examination, was a conventional melanocytic nevus, a Spitz nevus, or a combined melanocytic nevus. On the molecular level, one of the components revealed a mutation in the CTNNB1 gene encoding the $\beta$-catenin protein, while both components harbored a BRAF V600E mutation, without molecular features of a malignant melanocytic lesion. This presentation of a DPN of the lacrimal caruncle emphasizes the similarities of the caruncle with the skin.

(C) 2020 The Author(s)

Published by S. Karger AG, Basel

$\begin{array}{ll}\text { karger@karger.com } & \text { (c) 2020 The Author(s) } \\ \text { Pww.karger.com/oop } & \text { Published by S. Karger AG, Basel } \\ \text { This article is licensed under the Creative Commons Attribution- } \\ \text { NonCommercial-NoDerivatives 4.0 International License (CC BY- } \\ \text { NC-ND) (http://www.karger.com/Services/OpenAccessLicense). } \\ \text { Usage and distribution for commercial purposes as well as any dis- } \\ \text { tribution of modified material requires written permission. }\end{array}$




\section{Introduction}

Lesions located at the lacrimal caruncle are uncommon, and the differential diagnosis includes many different entities because the caruncle harbors both skin and conjunctival elements. Although the majority of lesions on this location is benign $[1,2]$, overlapping clinical and histopathological features make it challenging to distinguish the benign lesions from their malignant counterpart [2-4]. Therefore, thorough histological examination and additional diagnostic techniques for adequate management are required.

\section{Case Report}

An 18-year-old Caucasian female was referred to The Rotterdam Eye Hospital, Rotterdam, The Netherlands, because of a bleeding caruncular mass. She had a medical history of hyperpigmentation in the medial corner of the right eye for at least 6 years. On clinical examination, the caruncle of the right eye showed a $4-\mathrm{mm}$ stalked slightly asymmetrical dome-shaped pigmented hyperemic mass with crust and large vessels in the stalk (Fig. 1a). Vision was 1.20 for both eyes, with an intraocular pressure in the right eye and left eye of $16 \mathrm{~mm} \mathrm{Hg}$ and $11 \mathrm{~mm} \mathrm{Hg}$, respectively. For both diagnostic and therapeutic reasons the lesion was excised. The clinical differential diagnosis included pigmented papilloma, melanocytic nevus, melanoma, pyogenic granuloma, and oncocytoma.

Histological examination showed a papillomatous, partly pigmented melanocytic lesion with a junctional as well as a stromal component. The lesion was composed of two different components: one of which revealed a more nevoid character, with cells with scant amphophilic cytoplasm and a small hyperchromatic nucleus, and the other component showed a more epithelioid morphology, with cells with large dusty cytoplasm and a small, slightly irregular nucleus arranged in nests and fascicles (Fig. 1b, c). Moreover, perifollicular extension was found. There was no obvious cytological atypia, yet one mitotic figure was seen in the stromal part of the lesion. The conventional nevoid component showed maturation, in contrast to the epithelioid component. Furthermore, associated melanophages were seen. Using immunohistochemistry, both components showed diffuse expression of Melan A. HMB-45 showed loss of expression in the nevoid component, while expression remained in the epithelioid component (Fig. 1f). Both components showed positivity for BRAF V600E (Fig. 1g) and no loss of expression of BAP1 or p16. Staining for $\beta$-catenin (Fig. 1d) showed, mainly in the epithelioid component and to a lesser extent in the nevoid component in both the upper as well as the lower part of the lesion, extensive cytoplasmic and membranous expression, but no nuclear expression. Additionally, Cyclin D1 (Fig. 1e) showed a strong nuclear staining in the epithelioid component,
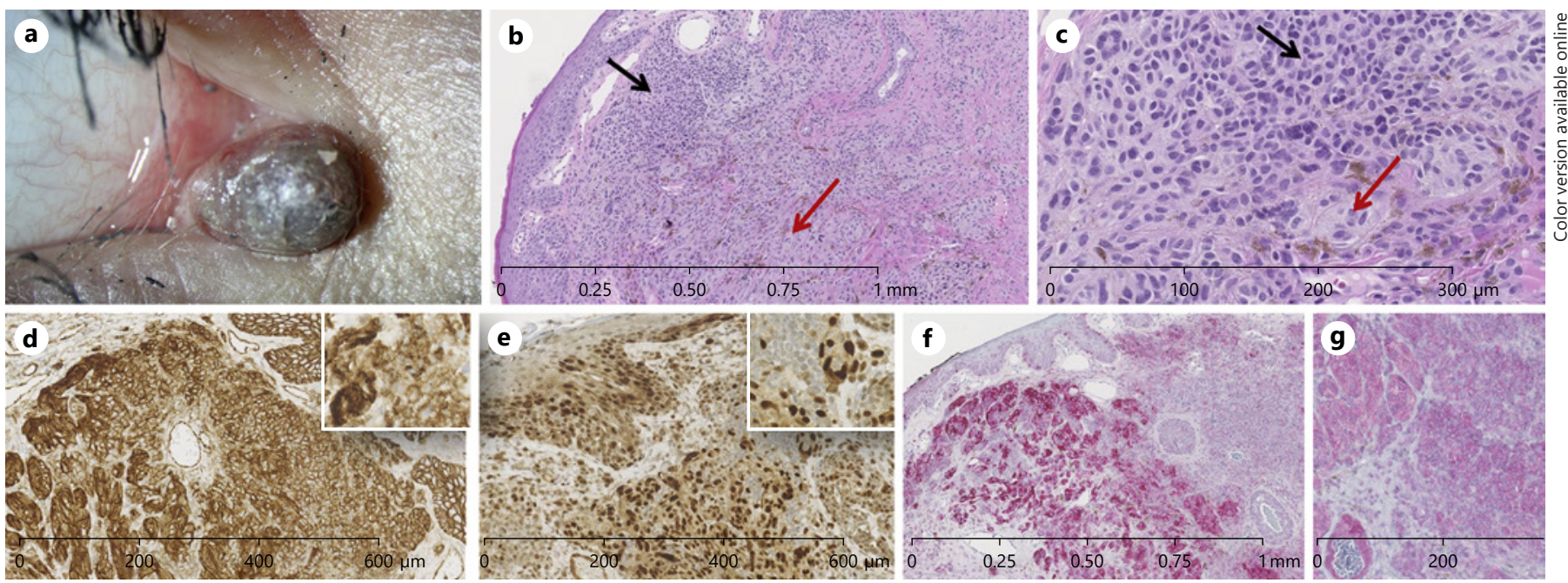

Fig. 1. Macroscopic view, $H \& E$ and immunohistochemical staining of the DPN. Photograph demonstrating a 4-mm slightly asymmetrical dome-shaped pigmented lesion located on the caruncle (a). The H\&E staining shows both the nevoid component (upper part) and the epithelioid component (lower part) at magnifications of $5 \times(\mathbf{b})$ and $20 \times(\mathbf{c})$, with the black and red arrow demonstrating the nevoid component and the epithelioid component, respectively. The epithelioid component is arranged in compact nests of cells with large dusty cytoplasm and small, slightly irregular nucleus. These nests show some pigmentation. In contrast, the nevoid component consists of loosely arranged clusters of cells with scant am- phophilic cytoplasm and a small hyperchromatic nucleus. The immunohistochemical stainings show absence of nuclear expression of $\beta$-catenin (magnification $10 \times$, with a magnification of $40 \times$ in the upper right corner; d), strong nuclear expression in the epithelioid component, and absent to weak expression of Cyclin D1 in the deeper parts of the nevoid component (magnification $5 \times$, with a magnification of $40 \times$ in the upper right corner; e), and dark staining of the epithelioid component in contrast to the nevoid component in the HMB-45 staining (magnification $5 \times$; f). Both components show immunohistochemical expression of BRAF V600E (magnification $10 \times ; \mathbf{g}$ ). 
with an absent to weak staining in the deep parts of the nevoid component. Triple fluorescence in situ hybridization showed no abnormalities for CCND1, MYB, and RREB1.

The two components of the lesion were isolated from the formalin-fixed paraffin-embedded material and were analyzed separately for molecular analysis. Both components showed a mutation in BRAF exon 15:c.1799 T>A; p.V600E, using mutation-specific PCR. Furthermore, targeted next-generation sequencing analysis confirmed this $B R A F$ mutation and revealed a mutation in CTNNB1 exon 3:c.134 C>T; p.S45F in the epithelioid component in contrast to the conventional nevoid component (Fig. 2). There were no GNA11, GNAQ, GNAS, HRAS, NRAS, APC, MAP2K1, and TERT promoter mutations, and there was no loss of CDKN2A. No additional copy number variations were identified by SNP array. Altogether, these findings are consistent with a deep penetrating nevus (DPN).

\section{Discussion/Conclusion}

Lesions located on the lacrimal caruncle are rare, with $10 \%$ comprising (pre-)malignant lesions. Over $40 \%$ of the lesions that present on the caruncle are pigmented [2].
Although the majority of the pigmented lesions are conventional melanocytic nevi $[1,2]$, the (pre-) malignant melanocytic lesions warrant special attention. Distinguishing benign lesions from their malignant counterpart can be very challenging [2-4], both from a clinical and histological point of view, because of various overlapping features. The caruncle comprises both skin and conjunctival elements. Therefore, lesions of both tissue types must be considered in the differential diagnosis. In the skin, melanocytic lesions comprise a broad differential diagnosis, including a conventional melanocytic nevus [5], a blue nevus, a Spitz nevus, a DPN, and melanoma. $[5,6]$. The difference between melanoma and a DPN can be very challenging as both may share worrisome features including cytological atypia, mitotic figures in the deeper parts of the lesion, and absent maturation. Furthermore, a DPN may show perineural extension. Yet, in contrast to melanoma, in DPN, recurrences are uncommon [4] and these lesions rarely show malignant transformation $[4,7]$. This emphasizes the need for additional techniques, in-

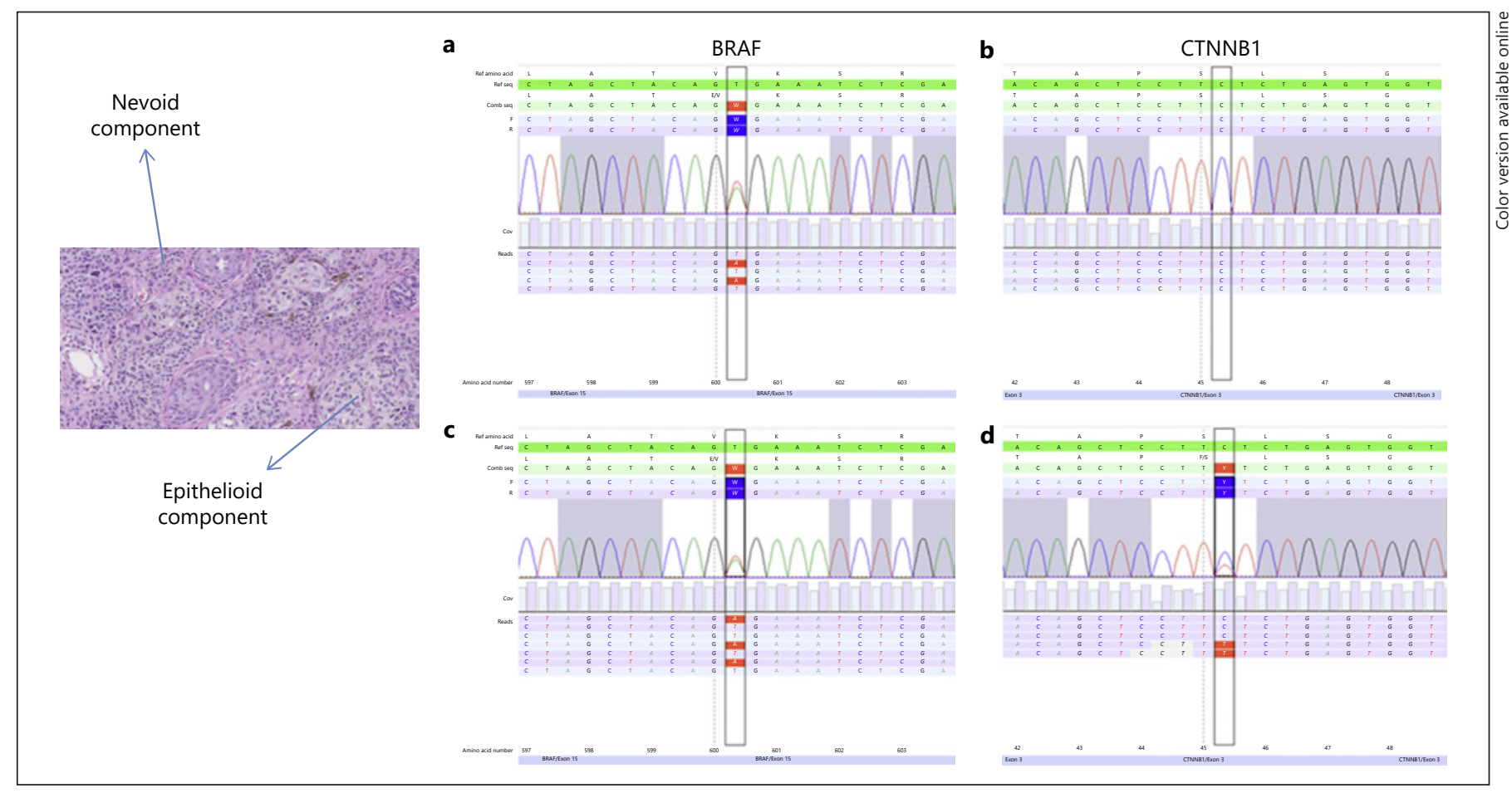

Fig. 2. Targeted next-generation sequencing concerning mutations in the BRAF and CTNNB1 gene. Targeted next-generation sequencing analysis revealed a missense mutation in the $B R A F$ gene, comprising a substitution at codon 600 (NM_004333; c.1799 $\mathrm{T}>\mathrm{A})$, resulting in a change from amino acid valine $(\mathrm{V})$ to glutamic acid (E) (p.V600E) in both the nevoid component and the epithelioid component (a, c, respectively). In contrast to the nevoid component (b), the epithelioid component (d) also harbored a missense mutation in the CTNNB1 gene encoding the $\beta$-catenin protein, comprising a substitution at codon 45 (NM_001098209; c.134 $\mathrm{C}>\mathrm{T}$ ), resulting in a change from amino acid serine $(\mathrm{S})$ to phenylalanine (F) (p.S45F). Ref, reference; seq, sequence; comb, combined; F, forward; R, reverse; Cov, coverage. 
cluding immunohistochemistry and molecular analysis, for further characterization.

The current lesion harbored two different components with presence of a mitotic figure in the stromal part of the lesion and one of the components lacking maturation, without loss of staining intensity in the HMB- 45 staining towards the deeper parts of the lesion. Additional to these worrying features, both components harbored a $B R A F$ mutation, which may occur in both skin and conjunctival melanoma $[7,8]$. In this perspective, $\beta$-catenin, with direct transcriptional target cyclin D1 [9], is of interest, which plays an important role in both the MAPK pathway [7] and the Wnt pathway [3]. A $\beta$-catenin mutation is absent in conventional nevi, but is present in DPNs [7]. Although this mutation is also found in DPN-like melanoma, both skin and conjunctival melanoma harbor TERT mutations $[7,10]$. Moreover (DPN-like) skin melanomas are reported to also harbor TP53 and BAP1 mutations, as well as biallelic loss of $C D K N 2 A$, in contrast to DPN without malignant behavior [7]. Since these mutations were not detected in our case, the diagnosis of DPN is confirmed.

In conclusion, this is the first description, including molecular characterization, of a DPN located on the lacrimal caruncle. Because of the unique composition of the caruncle, there is a broad differential diagnosis regarding pigmented lesions. As these lesions may be very difficult to discriminate by clinical and histological examination only, additional molecular analysis might be necessary in rendering a correct diagnosis. Furthermore, this first presentation of a DPN of the lacrimal caruncle emphasizes the similarities of the caruncle with the skin.

\section{Acknowledgement}

Thanks to J. Giang, MD, for her dedication to this case. Thanks to F. Groenendijk, MD, PhD, for his help with the molecular analysis.

\section{Statement of Ethics}

This research was conducted ethically in accordance with the World Medical Association Declaration of Helsinki and the "Code of Conduct for Responsible Use of Human Tissue and Medical Research" that applies to medical research in The Netherlands.

\section{Disclosure Statement}

The authors have no conflicts of interest to declare.

\section{Funding Sources}

This analysis was conducted as a part of routine diagnostics. No additional funding sources were required.

\section{Author Contributions}

All authors cooperated in collection and interpretation of the data, reviewed the manuscript critically, and agreed upon publishing the final version.

\section{References}

1 Levy J, Ilsar M, Deckel Y, Maly A, Pe'er J. Lesions of the caruncle: a description of 42 cases and a review of the literature. Eye (Lond). 2009 May;23(5):1004-18.

2 Verdijk RM, Luschyk T, Weijtens O, Van den Bosch W, Paridaens D. Survey of the histopathological and clinical characteristics of caruncular lesions; lessons from a retrospective case series over 25 years completed with an extensive review of literature. Available from: demo.webmicroscope.net/Handouts/ HO_93_Handout_ESP_2013_Ophthalmic_ Pathology_Verdijk.pdf

3 Reddy HS, Keene CD, Chang SH, Jian-Amadi A, Cimino PJ. Immunohistochemical profiling including beta-catenin in conjunctival melanocytic lesions. Exp Mol Pathol. 2017 Apr;102(2):198-202.
4 Luzar B, Calonje E. Deep penetrating nevus: a review. Arch Pathol Lab Med. 2011 Mar; 135(3):321-6.

5 de la Fouchardière A, Caillot C, Jacquemus I, Durieux E, Houlier A, Haddad V, et al. $\beta$-Catenin nuclear expression discriminates deep penetrating nevi from other cutaneous melanocytic tumors. Virchows Arch. 2019 May;474(5):539-50.

6 Bender RP, McGinniss MJ, Esmay P, Velazquez EF, Reimann JD. Identification of HRAS mutations and absence of GNAQ or GNA11 mutations in deep penetrating nevi. Mod Pathol. 2013 Oct;26(10):1320-8.

7 Yeh I, Lang UE, Durieux E, Tee MK, Jorapur A, Shain AH, et al. Combined activation of MAP kinase pathway and $\beta$-catenin signaling cause deep penetrating nevi. Nat Commun. 2017 Sep;8(1):644.
8 Cao J, Heijkants RC, Jochemsen AG, Dogrusöz M, de Lange MJ, van der Velden PA, et al. Targeting of the MAPK and AKT pathways in conjunctival melanoma shows potential synergy. Oncotarget. $2016 \mathrm{Jul} ; 8$ (35): 58021-36.

9 Shtutman M, Zhurinsky J, Simcha I, Albanese C, D'Amico M, Pestell R, et al. The cyclin D1 gene is a target of the beta-catenin/LEF-1 pathway. Proc Natl Acad Sci USA. 1999 May; 96(10):5522-7.

10 Koopmans AE, Ober K, Dubbink HJ, Paridaens D, Naus NC, Belunek S, et al.; Rotterdam Ocular Melanoma Study Group. Prevalence and implications of TERT promoter mutation in uveal and conjunctival melanoma and in benign and premalignant conjunctival melanocytic lesions. Invest Ophthalmol Vis Sci. 2014 Aug;55(9):6024-30. 